EXTENDED REPORT

\title{
Probing glaucoma visual damage by rarebit perimetry
}

\author{
P Brusini, M L Salvetat, L Parisi, M Zeppieri
}

Br J Ophthalmol 2005;89:180-184. doi: 10.1136/bjo.2003.041178

See end of article for authors' affiliations .....................

Correspondence to: P Brusini, Department of Ophthalmology, S Maria della Misericordia Hospital, Udine, Italy; prim.oculistica@aoud. sanita.fvg.it

Accepted for publication 26 July 2004

\begin{abstract}
Aim: To compare rarebit perimetry (RBP) with standard achromatic perimetry (SAP) in detecting early glaucomatous functional damage.

Methods: 43 patients with ocular hypertension $(\mathrm{OH}), 39$ with early primary open angle glaucoma (POAG), and 41 controls were considered. Visual fields were assessed using the Humphrey field analyser (HFA) 30-2 and RBP tests. Differences among the groups were evaluated using Student-Newman-Keuls and $\chi^{2}$ tests. Correlation between HFA and RBP parameters was assessed using the Pearson's correlation coefficients and regression analysis. Sensitivity and specificity of RBP in detecting early glaucomatous visual damage were calculated with different algorithms.

Results: RBP-mean hit rate (MHR) was respectively $88.6 \%$ (SD 4.8\%) in controls; $79.1 \%(10.9 \%$ ) in the $\mathrm{OH}$ group; $64.3 \%(13.8 \%)$ in the POAG group (differences statistically significant). Good correlation in the POAG group was found between HFA-mean deviation and RBP-MHR. Largest AROC (0.95) and optimal sensitivity (97.4\%) were obtained when an abnormal RBP test was defined as having (at least 1): MHR $<80 \%$; $>15$ areas with a non-hit rate of $>10 \%$; $\geqslant 2$ areas with a non-hit rate of $>50 \%$; at least one area with a non-hit rate of $\geqslant 70 \%$.

Conclusions: The RBP appeared to be a rapid, comfortable, and easily available perimetric test (requiring only a PC device), showing a high sensitivity and specificity in detecting early glaucomatous visual field defects.
\end{abstract}

G laucoma is one of the most common eye diseases, affecting about $2 \%$ of the population older than 50 years. ${ }^{1}$ It is characterised by progressive optic nerve damage $^{2}$ and retinal ganglion cells loss. ${ }^{3}$ Perimetry is considered the fundamental method in the diagnosis and follow up of glaucoma.

Previous studies have demonstrated that the standard achromatic perimetry (SAP), considered as the gold standard in visual field (VF) examination, cannot detect damage until about $20-50 \%$ of the ganglion cells have been lost, ${ }^{2-6}$ and that the optic disc and retinal nerve fibre layer (RNFL) alterations, caused by ganglion cell axons loss, commonly occur before the perimetric defects. ${ }^{7}$

In order to detect VF damage before it can be seen with SAP, other perimetric techniques have been developed including frequency doubling technology (FDT), ${ }^{8}$ motion perimetry, ${ }^{9}$ flicker perimetry, ${ }^{10}$ high pass resolution perimetry (HRP), ${ }^{11}{ }^{12}$ and short wavelength automated perimetry (SWAP). ${ }^{13}$

Rarebit perimetry (RBP) is a new perimetric method, developed by Frisén, ${ }^{14}$ which has given promising preliminary results in the early detection of VF damages in patients with neurological disorders. ${ }^{14}{ }^{15}$ As far we know, RBP use on glaucomatous patients has yet to be reported in literature.

In this consecutive comparative observational study, the SAP and RBP data of patients with ocular hypertension $(\mathrm{OH})$ or early primary open angle glaucoma (POAG), and of controls, were compared and discussed.

\section{METHODS}

The study included 43 patients affected with $\mathrm{OH}, 39$ patients with early POAG, and 41 age matched controls. After obtaining informed consent, all subjects underwent an ophthalmological examination (including best corrected visual acuity (BCVA) evaluation, slit lamp examination, Goldmann applanation tonometry, gonioscopy, fundus biomicroscopy). VF examination with SAP and RBP was then performed within 1 month. One eye per patient was randomly selected for analysis, except when only one eye met our inclusion criteria.

Inclusion criteria were age range of 35-72 years, BCVA $\geqslant 0.8$, open anterior chamber angle, absence of ocular pathology other than glaucoma, mild nuclear sclerosis, and rare drusen.

Exclusion criteria included ametropia more than plus or minus 5 dioptres, pupils diameter $<3 \mathrm{~mm}$, anterior angle alterations, presence of secondary causes of glaucoma, history of intraocular surgery, diabetes mellitus, neurological disorders, and medications altering the VF.

Controls were screened to have a normal intraocular pressure (IOP) and to exclude glaucoma family history and ocular pathologies. VF were assessed with SAP using the Humphrey field analyser (HFA) II 750 (Carl Zeiss Meditec Inc, Dublin, CA, USA) program 30-2, ${ }^{16}$ with Swedish Interactive Threshold Algorithm (SITA) strategy.

SAP tests were classified as glaucomatous according to the Anderson criteria, ${ }^{17}$ in which at least one of the following was present:

(1) a cluster of $\geqslant 3$ points in the pattern deviation probability plot, located in areas typically observed in glaucoma, having a probability level of $<5 \%$, with at least one point having a probability level of $<1 \%$; none of the points could be edge points unless they were located immediately above or below the nasal horizontal meridian;

(2) PSD probability level of $<5 \%$;

(3) glaucoma hemifield test outside normal limits.

Abbreviations: BCVA, best corrected visual acuity; FDT, frequency doubling technology; HFA, Humphrey field analyser; HRP, high pass resolution perimetry; IOP, intraocular pressure; $M D$, mean deviation; MHR, mean hit rate; $\mathrm{OH}$, ocular hypertension; $\mathrm{POAG}$, primary open angle glaucoma; PSD, pattern standard deviation; RBP, rarebit perimetry; RNFL, retinal nerve fibre layer; SITA, Swedish Interactive Threshold Algorithm; SWAP, short wavelength automated perimetry; VF, visual field 
Reliable criteria for HFA tests included false positive and false negative responses of $<33 \%$ and fixation losses of $<20 \%$. Glaucomatous VF defects were classified using the Glaucoma Staging System, ${ }^{18}$ which classifies severity in five stages. Stages 0,1 , and 2 were considered, which included tests having a mean deviation $(\mathrm{MD})$ of $>-9.0$ and a pattern standard deviation (PSD) of $<8.0 \mathrm{~dB} .{ }^{18}$

Optic disc and RNFL appearance were analysed by an expert ophthalmologist with slit lamp biomicroscopy using a $+78 \mathrm{D}$ Volk lens, and classified utilising the European Glaucoma Society (EGS) guidelines ${ }^{19}$ (table 1).

All eyes having both an IOP $>21 \mathrm{~mm} \mathrm{Hg}$ and normal SAP results were evaluated with a scanning laser polarimeter having a variable corneal compensator (GDx-VCC) (Laser Diagnostic Technologies, Inc, San Diego, CA, USA). ${ }^{20}$ The GDx images were judged by an expert ophthalmologist based on the software provided parameters.

The patients were classified into three groups according to the EGS criteria ${ }^{19}$ :

(1) $\mathrm{OH}$ group (43 eyes): IOP $>21 \mathrm{~mm} \mathrm{Hg}$; optic disc and RNFL appearance, and SAP results all normal;

(2) POAG group (39 eyes): IOP $>21 \mathrm{~mm} \mathrm{Hg}$ before medications, typically glaucomatous optic disc or RNFL changes, either with reproducible glaucomatous SAP VF defect (perimetric POAG, 33 eyes) or without (pre-perimetric POAG, six eyes);

(3) control group (4l eyes): IOP, optic disc and RNFL appearance and SAP results all normal.

The RBP procedure has already been described elsewhere. ${ }^{14}$ In brief, the RBP test is performed on any standard PC with a liquid crystal display; the software (in Microsoft Windows format) is available free of charge from the author.

The test stimulus is composed of two microdots, having a diameter set at half of the minimum angle of resolution (approximately 1/100 the size of the SAP stimulus), separated by $4^{\circ}$ of the visual angle and simultaneously exposed for $200 \mathrm{~ms}$. Paired dots appear on the screen at random positions within a $5^{\circ}$ circular diameter area centred on each of four central and 26 peripheral test locations. A total of 30 areas, separated by $10^{\circ}$ (centre to centre), are tested, covering a horizontal eccentricity of $27.5^{\circ}$ and a vertical eccentricity of $20^{\circ}$ upwards and of $22.5^{\circ}$ downwards (fig 1 ). The tested area distribution is the same for both the right and left eye.

The first test phase involves one pass $(=2$ microdots presentation) over each of the tested areas; a minimum of five runs is recommended, for a total of 10 presentations per area; $10 \%$ of presentations, containing either only one dot or none at all, are used as a control. Target and background luminances are set at $150 \mathrm{~cd} / \mathrm{m}^{2}$ and $1 \mathrm{~cd} / \mathrm{m}^{2}$ respectively. The room illumination required is 1 lux.

A fixation mark is moved at in a preset sequence by the computer. Subjects are instructed to fixate it on the monitor, to indicate the number of microdots seen during each

Table 1 Glaucomatous characteristics of optic disc and retinal nerve fibre layer (RNFL) as stated by the European Glaucoma Society (at least 1)

(1) Optic disc excavation - that is, undermining of the neural rim

(2) Notching involving $\geqslant 2$ clock hours

(3) Focal or diffuse atrophy of neural rim area involving $\geqslant 2$ clock hours

(4) Vertical cup to disc ratio $>0.6$

(5) Cup to disc asymmetry between fellow eyes $>0.2$

(6) Disc haemorrhage

(7) Baring of circumlinear blood vessels

(8) Focal or generalised atrophy of the RNFL.

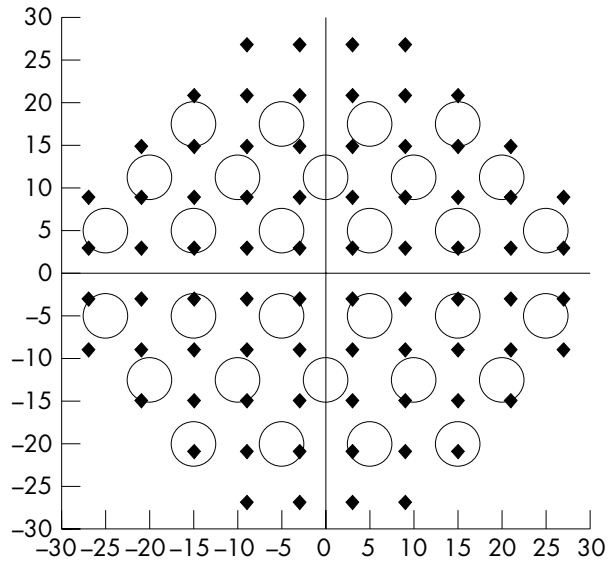

Figure 1 Representation of size and distribution of the 30 test areas in rarebit perimetry with the corresponding test points used in the Humphrey field analyser program 30-2.

presentation $(0,1$, or 2$)$ by not clicking, clicking, or double clicking a mouse button, and to refrain from gazing at eccentric stimuli.

The test was first performed at 0.5 metres of distance, to test the 26 peripheral areas, and repeated at a distance of 1 metre, for the four central locations. ${ }^{21}$

Total refractive error and proper test distance were corrected with the spherical equivalent. The RBP results are provided as a hit rate, expressing the total number of dots seen divided by the total number of dots shown, calculated as a percentage. The printout provides a mean hit rate (MHR), which is calculated over all the tested locations (except for the one closest to the blind spot). 30 non-hit rate values, plotted separately for each of the tested areas, are also provided.

The error statistic value represents the sum of the responses to control presentations, and it should come close to 0 . Reliability of RBP testing was defined as an error statistic value of $<2$. Left eye results were converted in a right eye format for the results analysis.

The following parameters were considered:

- for the HFA test: MD, PSD, testing time;

- for the RBP test: MHR, number of tested areas with a nonhit rate of 10 groups of misses (one group for each 10\% miss level), testing time, percentage of eyes having the following patterns of distribution of missed hit rates:

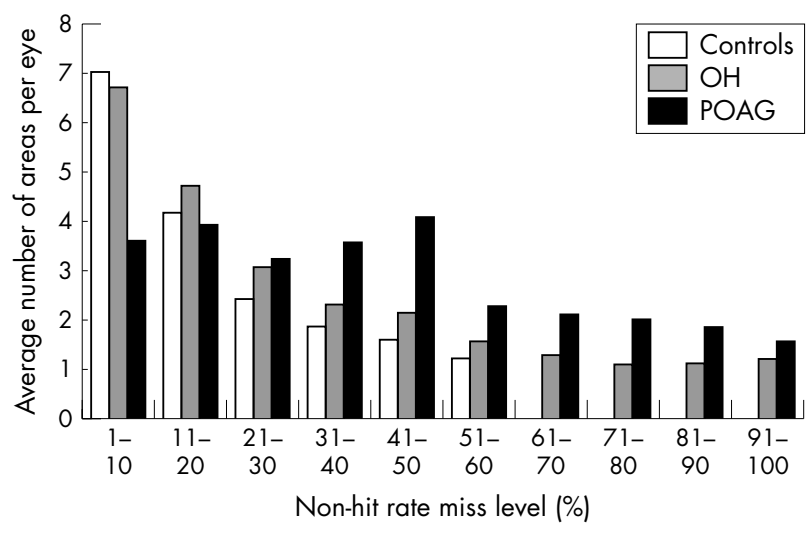

Figure 2 Histogram showing the mean number of areas per eye with a non-hit rate of 10 groups of misses (one group for each 10\% miss level) obtained by the three groups in the rarebit perimetry test. 


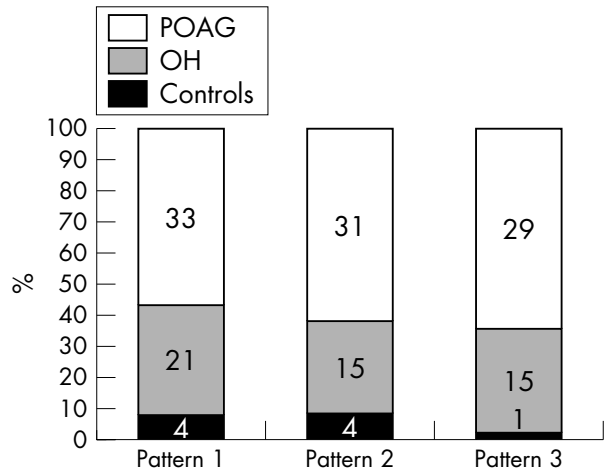

Figure 3 Histogram showing the number of eyes in the three groups having one of the following patterns of distribution of missed hit rates in the rarebit perimetry test: pattern 1: $\geqslant 2$ adjacent areas with a non-hit rate of $\geqslant 50 \%$, with at least one area with a non-hit rate of $>50 \%$; pattern 2: $\geqslant 3$ adjacent areas with a non-hit rate of $\geqslant 50 \%$, with at least one area with a non-hit rate of $>50 \%$; pattern 3 : $\geqslant 2$ adjacent areas with a non-hit rate of $>50 \%$

- pattern 1: $\geqslant 2$ adjacent areas with a non hit rate of $\geqslant 50 \%$, with at least one area with a non-hit rate of $>50 \%$;

- pattern 2: $\geqslant 3$ adjacent areas with a non-hit rate of $\geqslant 50 \%$, with at least one area with a non-hit rate of $>50 \%$;

- pattern 3: $\geqslant 2$ adjacent areas with a non-hit rate of $>50 \%$.

Sensitivity (Se), specificity (Sp), and area under the receiver operating characteristic (ROC) curves (AROC) of RBP in detecting glaucoma visual damage were calculated using different algorithms. The HFA 30-2 test and the evaluation of optic disc and RNFL, utilising both slit lamp biomocroscopy and GDx VCC images, were considered as the gold standard. Differences between the HFA and RBP results for the groups were evaluated using Kruskal-Wallis, StudentNewman-Keuls ( $\mathrm{S}-\mathrm{N}-\mathrm{K})$, and $\chi^{2}$ tests. Correlation between HFA-MD and RBP-MHR parameters was calculated using Pearson's correlation coefficients and regression analysis. Differences among AROCs were evaluated using the Hanley and McNeil method. ${ }^{22}$ Difference between the average test time of HFA and RBP was tested using the Student's $t$ test. A $p$ value of $<0.05$ was taken as statistically significant.

\section{RESULTS}

The control subjects were significantly younger than patients with both $\mathrm{OH}$ and POAG (Kruskal-Wallis test, $\mathrm{p}<0.02$, table 2).

The average HFA-MD and HFA-PSD values were significantly higher in the POAG than in control and $\mathrm{OH}$ eyes ( $\mathrm{S}-\mathrm{N}-\mathrm{K}$ test, $\mathrm{p}<0.01$, table 2). The mean RBP-MHR of control, $\mathrm{OH}$, and POAG groups was respectively $88.6 \%$ (SD 4.8\%) (range 78 $98 \%), 79.1 \%(10.9 \%)$ (range $44-95 \%)$, and $64.3 \%(13.8 \%)$ (range $37-96 \%$ ) (and $71.2 \%$ (10.4\%), range $54-81 \%$, in the

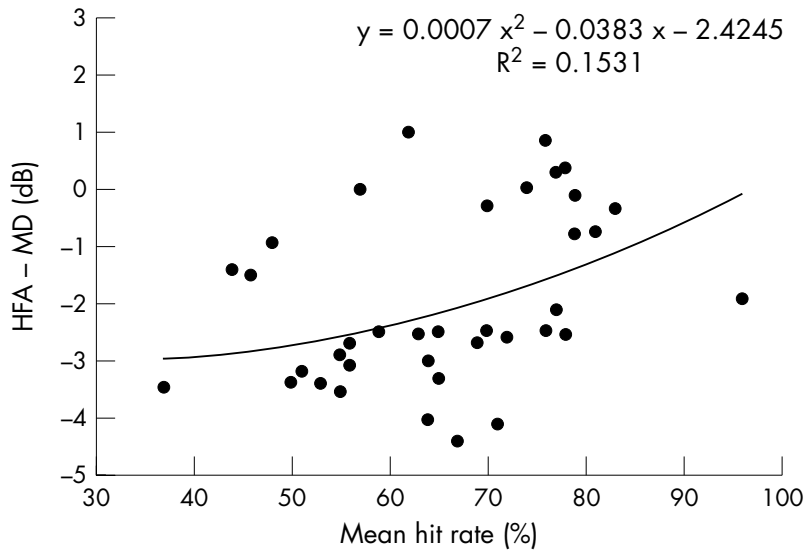

Figure 4 Scatter plot illustrating the relation between Humphrey field analyser-mean deviation (HFA-MD) and rarebit perimetry-mean hit rate in the glaucomatous eyes.

pre-perimetric-POAG subgroup), with statistically significant differences among the groups $(\mathrm{S}-\mathrm{N}-\mathrm{K}$ test, $\mathrm{p}<0.01)$. Significant reduction of the RBP-MHR was observed as age increased in controls, with a mean age decline of $0.15 \%$ per year.

As shown in figure 2, in individual cases one or more misses $>10 \%$ occurred on average in 9.0 (4.2) tested areas (range 1-21) in controls; 14.2 (5.5) areas (range 2-29) in the $\mathrm{OH}$ group; and $21.2(5.7)$ areas (range 3-29) in the POAG group (19.8 (5.2), range 14-28, in the pre-perimetric POAG patients). Differences among the groups were statistically significant ( $\mathrm{S}-\mathrm{N}-\mathrm{K}$ test, $\mathrm{p}<0.01)$.

Areas with a non-hit rate between $11 \%$ and 30\% were equally distributed within the groups; areas with a non-hit rate between $31 \%$ and $60 \%$ were significantly more frequent in the POAG group; areas with a non-hit rate of $>60 \%$ appeared significantly less in the controls and more in the POAG group ( $\mathrm{S}-\mathrm{N}-\mathrm{K}$ test, $\mathrm{p}<0.01$ ).

The percentage of eyes having at least one of the patterns of distribution of missed hit rates were the lowest in the controls $(9.8 \%)$, intermediate in the $\mathrm{OH}(48.8 \%)$, and the highest in the POAG eyes $(84.6 \%)$, showing significant differences among the groups for all of the pattern types $\left(\chi^{2}\right.$ test, $\mathrm{p}<0.01$, fig 3 ).

Significant correlation was found between HFA-MD and RBP-MHR in the POAG groups (Pearson's $r$ correlation coefficient of $0.38, \mathrm{p}<0.02$ ). The scatter plot comparing the HFA-MD with the RBP-MHR for each eye of the POAG group, with an adapted regression curve, showed a trend towards a greater abnormality in the HFA-MD as RBP-MHR decreased, and a tendency of obtaining higher measured values using RBP (fig 4).

As listed in table 3, the Se of the RBP using the different algorithms ranged from $82.1 \%$ to $97.4 \%$. Sp ranged from $90.2 \%$ to $100 \%$. The AROC values ranged from 0.89 to 0.95 (differences not statistically significant, Hanley and McNeil method).

Table 2 Patient demographics and Humphrey field analyser (HFA) data

\begin{tabular}{|c|c|c|c|c|}
\hline \multirow[b]{2}{*}{ Groups (no of eyes) } & \multirow{2}{*}{$\frac{\text { Age (years) }}{\text { Mean (SD) }}$} & \multirow[b]{2}{*}{$M / F$} & \multirow{2}{*}{$\frac{M D}{\text { Mean (SD) }}$} & \multirow{2}{*}{$\frac{\text { PSD }}{\text { Mean (SD) }}$} \\
\hline & & & & \\
\hline $\begin{array}{l}\text { Controls (41) } \\
\text { OH (43) } \\
\text { POAG (39) }\end{array}$ & $\begin{array}{l}54(9.0) \text { (range 35-72) } \\
61(8.1) \text { (range 36-72) } \\
61(10.1) \text { (range 36-72) }\end{array}$ & $\begin{array}{l}13 / 28 \\
15 / 28 \\
19 / 20\end{array}$ & $\begin{array}{l}-0.4(1.2) \\
-0.8(1.2) \\
-2.0(1.6)\end{array}$ & $\begin{array}{l}1.6(0.2) \\
1.6(0.3) \\
3.1(1.4)\end{array}$ \\
\hline
\end{tabular}

$\mathrm{OH}$, ocular hypertension; POAG, primary open angle glaucoma; SD, standard deviation; $M D$, mean deviation; PSD, pattern standard deviation. 
Table 3 Evaluation of different algorithms for discriminating between controls and glaucomatous eyes using the rarebit perimetry testing

\begin{tabular}{|c|c|c|c|c|}
\hline Algorithms & Sensitivity (\%) & Specificity (\%) & AROC $(\%)$ & $\begin{array}{l}\text { Abnormals in } \mathrm{OH} \text { group } \\
\text { (\% of eyes) }\end{array}$ \\
\hline (1) MHR of $<80 \%$ & 92.3 & 95.1 & 0.94 & 44.2 \\
\hline (2) $>15$ areas with a non-hit rate of $>10 \%$ & 87.2 & 97.6 & 0.92 & 51.2 \\
\hline$(3) \geqslant 2$ areas with a non-hit rate of $>50 \%$ & 84.6 & 95.1 & 0.90 & 44.2 \\
\hline (4) at least 1 area with a non-hit rate of $\geqslant 70 \%$ & 82.1 & 100 & 0.91 & 51.2 \\
\hline (5) positivity at 1 of the previous algorithms & 97.4 & 92.7 & 0.95 & 65.1 \\
\hline (6) positivity at 1 type of pattern distribution & 84.6 & 90.2 & 0.89 & 51.2 \\
\hline
\end{tabular}

Optimal Sp was found when the presence of "at least one area with a non-hit rate of $\geqslant 70 \%$ " was used as cut-off criterion.

Largest AROC and optimal Se were obtained when RBP tests were defined as abnormal if having at least one of the following: MHR $<80 \%$; $>15$ areas with a non-hit rate of $>10 \%$; $\geqslant 2$ areas with a non-hit rate of $>50 \%$; at least one area with a non-hit rate of $\geqslant 70 \%$.

The percentage of abnormal eyes tested with RBP in the $\mathrm{OH}$ group ranged from 44.2 to $65.1 \%$. The average RBP testing time was slightly, but not significantly, less (405 (49.3) seconds/patient) than SAP testing time (416 (54.8) seconds/patient).

\section{DISCUSSION}

The detection of early glaucomatous VF damage is of great clinical interest. Many new perimetric techniques have been developed with this purpose, ${ }^{8-13}$ one of the newest being the RBP. RBP utilises a spatially and temporally minimised test stimulus in order to avoid the simultaneous stimulation of many retinal receptive fields, with the risk of underestimating a defect.

The purpose of the RBP is to calculate the percentage of the visual system integrity by analysing the proportion of observed responses to microdots presentations. The basic concept that lies behind the RPB is that all normal eyes, although differing in the total number of ganglion cells, ${ }^{23}$ have a complete neuroretinal architecture, permitting the detection of pairs of dots of opportune size, contrast and separation, everywhere in the VF. A depleted neural matrix may give rise to the detection of just one or none of the target dots. ${ }^{14}$ Some misses can be expected on physiological grounds (the blind spot, angioscotomata, age related neuroretinal architecture depletion, blinks, attention lapses). ${ }^{24} 25$

In the present study, differences in MHR and non-hit rates within control, $\mathrm{OH}$, and POAG groups were evaluated in order to establish if these parameters could effectively reflect the functional integrity of the retinal ganglion cells; the pattern of distribution of areas with high non-hit rate was also investigated to find an abnormality criterion for RBP testing. The results obtained by our controls (mean RBPMHR of $88.61 \%(4.8 \%)$, with a decline with age averaging $0.15 \%$ year; one or more misses occurred in a mean of 9.0 (4.2) test areas (fig 3)), were slightly worse than those reported by Frisén in a cohort of slightly younger patients. ${ }^{14}$

The RBP-MHR progressively significantly decreased from the controls to the POAG group. Our controls were significantly younger than the glaucomatous patients. We believe, however, that this difference would not practically affect our results, since the age related reduction of RBPMHR was very small compared to the decrease induced by glaucoma.

The number and magnitude of missed hit rates appeared significantly lower in the controls and higher in the POAG group (fig 2). Adjacent areas having a high non-hit rate were more frequently observed in the $\mathrm{OH}$ and POAG groups rather than in the controls (fig 3). These results are interesting because our POAG group comprised only patients having either an early or pre-perimetric POAG.

The high number of abnormal RBP tests found in the $\mathrm{OH}$ (table 3) and in pre-perimetric POAG eyes, coupled with the tendency of observed higher values using RBP as apposed to SAP (fig 4), strongly suggests that RBP may depict a more advanced stage of glaucoma, improving the detection of low degrees of damage. This advantage in RBP can be attributed to various causes: the simplification of the target content; the replacement of the threshold procedure with a probe of the percentage of the visual system integrity; the possible lower inter-individual variability in the test results. ${ }^{14}$

The good correlation between HFA-MD and RBP-MHR in the glaucomatous eyes (fig 4) suggests that both values are probably related to the number of functioning retinal ganglion cells, and that counting the misses on the RBP test may determine the presence and severity of glaucoma.

Our best AROC value, of 0.95 , showing a Se and Sp respectively of $97.4 \%$ and $92.7 \%$ (table 3 ), indicates that the ability of RBP in differentiating between normal and early POAG is comparable, if not better, than that obtained by other non-conventional perimetries: in a cohort of mild to moderate glaucomatous patients, FDT showed a Se and Sp respectively of $93 \%$ and $100 \%{ }^{26}$; the HRP performances were respectively $82.5 \%$ and $85 \%,{ }^{27}$ and decreased to $61 \%$ and $90 \%$ with SWAP. ${ }^{28}$

Our patients did not experience any problem in performing $\mathrm{RBP}$, except for the advanced elderly, who were not familiar with the PC mouse; moreover, the RBP test appeared slightly faster than the HFA 30-2 SITA test. In conclusion, RBP appeared to be a rapid and easily administered perimetric test, able to detect early glaucomatous VF defects. With some improvements (a more accurate screen calibration, a designed patient head rest, a better fixation control), we think that RBP may become useful in both the screening and assessment of glaucoma and other neuro-ophthalmological pathologies, providing a quantitative VF testing method which is easily available in every medical office ( since it only requires a $\mathrm{PC}$ ), even when ordinary perimetry is impracticable-for example, at the bedside.

\section{Authors' affiliations}

P Brusini, M L Salvetat, L Parisi, M Zeppieri, Department of Ophthalmology, S Maria della Misericordia Hospital, Udine, Italy

\section{REFERENCES}

1 Ekstroem C. Prevalence of open-angle glaucoma in central Sweden. The Tierp Glaucoma Survey. Acta Ophthalmol Scand 1996;74:107-12.

2 Harwreth RS, Crauford ML, Frishman L, et al. Visual field defects and neural losses from experimental glaucoma. Prog Ret Eye Res 2002;21:91-125.

3 Quigley HA. Neuronal death in glaucoma. Prog Ret Eye Res 1999;18:39-57.

4 Quigley HA, Dunkelberger GR, Green WR. Retinal ganglion cell atrophy correlated with automated perimetry in human eyes with glaucoma. Am J Ophthalmol 1989;107:453-64. 
5 Kerringan-Baumrind LA, Quigley HA, Pease ME, et al. Number of ganglion cells in glaucoma eyes compared with threshold visual field tests in the same persons. Invest Ophthalmol Vis Sci 2000;41:741-8.

6 Curcio CA, Owsley C, Skalka HW, et al. Topography of retinal cells and visual sensitivity in the same human eye. Invest Ophthalmol Vis Sci 1993:34:777.

7 Pederson JE, Anderson DR. The mode of progressive disc cupping in ocular hypertension and glaucoma. Arch Ophthalmol 1980;58:14-19.

8 Cello KE, Nelson-Quigg JM, Johnson CA. Frequency doubling technology perimetry for detection of glaucomatous visual field loss. Am J Ophthalmol 2000;129:314-22.

9 Bosworth CF, Sample PA, Gupta N, et al. Motion automated perimetry identifies early glaucomatous field defects. Arch Ophthalmol 1998;116:1153-8.

10 Yoshiyama KK, Johnson CA. Which method of flicker perimetry is most effective for detection of glaucomatous visual field loss? Invest Ophthalmol Vis Sci 1997;38:2270-7.

11 Frisén L. High-pass resolution perimetry: evidence from parvocellular neural channel dependence. Neuro-ophthalmology 1992;12:257-64.

12 Chauhan BC, House PH, McCormick TA, et al. Comparison of conventional and high-pass resolution perimetry in a prospective study of patients with glaucoma and healthy controls. Arch Ophthalmol 1999;117:24-33.

13 Bayer AU, Maag KP, Erb C. Detection of optic neuropathy in glaucomatous eyes with normal standard visual fields using a test battery of short-wavelength automated perimetry and pattern electroretinography. Ophthalmology 2002;109:1350-61.

14 Frisén L. New, sensitive window on abnormal spatial vision: rarebit probing Vis Res 2002;42:1931-9.

15 Frisén L. Spatial vision in visually asymptomatic subjects at high risk for multiple sclerosis. J Neurol Neurosurg Psychiatry 2003;74:1145-47.

16 Heijl A. Humphrey field analyzer. In: Drance SM, Anderson DR, eds. Automated perimetry in glaucoma, a practical guide. Orlando: Grune and Stratton, 1985:129-40.
17 Anderson D, Patella V. Automated static perimetry. St. Louis: Mosby, 1999:117.

18 Brusini P. Clinical use of a new method for visual field classification in glaucoma. Eur J Ophthalmol 1996:6:402-7.

19 European Glaucoma Society. 2003 Terminology and guidelines for glaucoma. Savona, Italy: Dogma, 2003;ch 2, 5-8.

20 Reus NJ, Colen TP, Lemij HG, et al. Visualization of localized retinal nerve fiber layer defects with the GDx with individualized and with fixed compensation of the anterior segment birefringence. Ophthalmology 2003; 110:1512-16.

21 Frisén L. High-pass resolution perimetry: central-field neuroretinal correlates. Vis Res 1995;35:293-301.

22 Hanley A, McNeil BJ. A method of comparing the areas under the receiver operating characteristics curves derived from the same cases. Radiology 1983; 148:839-43.

23 Curcio CA, Allen KA. Topography of ganglion cells in human retina. J Comp Neurol 1990;300:5-25.

24 Frisén L. High-pass resolution perimetry and age-related loss of visual pathway neurons. Acta Ophthalmol 1991;69:511-15.

25 Schiefer U, Benda N, Dietrich TJ, et al. Angioscotomata detection with fundusoriented perimetry. A study with dark and bright stimuli of different sizes. Vis Res 1997;39:1897-909.

26 Johnson C, Samuels S. Screening for glaucomatous visual field loss with frequency-doubling perimetry. Invest Ophthalmol Vis Sci 1997;38:413-25.

27 Graham SL, Drance SM. Interpretation of high-pass resolution perimetry with a probability plot. Graefes Arch Clin Exp Ophthalmol 1995:233:140-9.

28 Sample PA, Bosworth CF, Blumenthal EZ, et al. Visual function-specific perimetry for indirect comparison of different ganglion cell populations in glaucoma. Invest Ophthalmol Vis Sci 2000;41:1783-90. 